\title{
Problems of Understanding Pulsars
}

Progress in pulsar theory is slower than might be expected from the wealth of observational data now available. An important step forward was the statement by Goldreich and Julian in 1969 (Astrophys. J., 157, 869) that a rotating, magnetized neutron star must be surrounded by a very remarkable co-rotating magnetosphere, in which gravity counts for almost nothing and only electrodynamic forces determine the particle motion out to a distance where the co-rotation velocity approaches the velocity of light. Mestel (Nature Physical Science, 233,$149 ; 1971$ ) has recently given a more general account of this theory. But astronomers seem to be no nearer to an understanding of the basic radiation process. If anybody claims to understand pulsars, let him answer the question "How do they pulse?"

What is needed is a twin attack from observation and from theory. A co-rotating magnetosphere provides a fairly straightforward analytical problem, provided that the particle density is so low that it does not affect the magnetic field. This is almost certainly not the case in the interesting region close to the velocity of light circle, but there are other problems extending throughout a more realistic magnetosphere. A self-consistent solution including a full complement of particles and magnetic field must face problems such as the following.

- Do the particles gain and radiate most of their energy close to the pulsar surface or near the velocity of light circle?

What are the particle trajectories near the velocity of light circle?

Is there a complete charge separation in clearly defined regions of the magnetosphere?

Is the charge of the outward electron flow balanced by a return flow of electrons or by an outward flow of protons?

What is the mechanism for bunching which leads to coherent radio emission?

Observation may provide some help in describing the geometry of the field and the electron trajectories in the emitting region. But to date there is no agreement about the location of the emitting region, and no clear interpretation of the polarization measurements which should provide a description of the magnetic field. There should be some critical experiments which could provide a real guide to the field configuration, but none has been forthcoming so far.

Another approach is to consider how the pulsar magnetosphere might evolve as the spin rate decreases. An interesting way out of the analytical problem then emerges: old pulsars might not have a dense magnetosphere at all. Ruderman (Phys. Rev. Lett., 27, 1306 ; 1971) has suggested that slow rotation provides such a small surface field that no electrons are emitted. Thermionic emission is unlikely, so the magnetosphere may be empty. This would certainly account for the dearth of pulsars with periods greater than $2 \mathrm{~s}$.

Endean carries this argument further in an article on page 184 of this issue of Nature by attempting to put the observational data on young and old pulsars into some sort of evolutionary sequence. First attempts at this will undoubtedly be shot down by observers who are in possession of so much detailed material that they can shoot down almost any interpretation. But the chief point is that a theorist who has contributed to the analysis of the magnetospheric problem is now in touch with the observations. There is a particular need for an explanation of the pattern of drifting sub-pulses, which seem to be characteristic of the older pulsars. Endean points out that this may fit the evolutionary stage just before the magnetosphere becomes a vacuum. It also fits the hypothesis that the emission takes place from close to the velocity of light cylinder.

There is still some way to go before speculation of this kind can give way to a solid theory of the pulsar magnetosphere, but it is encouraging to see speculation which takes some account of the observational facts.-F. G. S.

\section{Miocene-Pliocene Boundary}

FIJI is odd, for it contains both continental and oceanic rocks, but the concept of world-wide stratigraphical boundaries is even odder. Both are, however, critical to an understanding of the evolution of the Earth and are brought together by Gill and McDougall on page 176 of this issue of Nature. The oddity of stratigraphical boundaries is that they are purely conceptual phenomena, originally used in the eighteenth century to distinguish and relate one sequence of rocks to another. As such they usually reflected some particular facies of sedimentation, the boundaries being marked by either a significant change in the nature of the rocks deposited or, more commonly, by a distinct break in sedimentation during which the underlying rocks were often tilted or folded and then partially eroded before deposition of the next sequence.

Clearly, the recognition of such lithostratigraphical intervals is essential for unravelling the geological history of any one area, but problems arise when attempts are made to trace such boundaries into neighbouring areas. This is because the lithological unit clearly represents a specific interval of geological time during which that particular rock type was deposited whereas the boundaries also represent periods of geological time. Thus the boundaries of such units may, if they are recognizable at all, occur at significantly different times in closely neighbouring areas.

During the Phanerozoic, the past 550 million years, there was a great increase in the types and preservability of organisms. This has allowed the definition of time intervals at that time during which certain organisms lived. In 1810 Brongniart defined the Tertiary period as that interval during which the rocks overlying the Cretaceous Chalk of the Paris Basin were laid down; this is the 